\title{
Schulabsentismus und die Bedeutung von Schule und Familie
}

\section{Christine Sälzer ${ }^{1}$}

Dass Schulabsentismus ein Ergebnis von multifaktoriellen Bedingungsgefügen ist, gilt inzwischen als unbestritten. Wenn Jugendliche ihrer Schulpflicht nicht nachkommen, werden in der Regel deren Eltern zur Verantwortung gezogen; auf der anderen Seite nimmt jedoch die Schule eine wichtige Funktion für den regelmässigen Schulbesuch ein. In diesem Artikel wird anhand von Mehrebenenanalysen untersucht, inwieweit familiäre und schulische Merkmale zusammenwirken, wenn man die unterschiedlichen Häufigkeiten von Schulabsentismus vom gelegentlichen bis zum massiven Schwänzen betrachtet. Es zeigt sich, dass sowohl Familie als auch Schule relevant für das Absentismusverhalten sind, wobei die Reaktionen der Eltern auf Schulschwänzen die deutlichsten Effekte zeigen.

\section{Einleitung}

«Du hast Pickel im Gesicht. Und kein Bock auf gar nichts. Deine Fehlstunden nähern sich mittlerweile dem dreistelligen Bereich. Deine Haare werden immer länger und deine Hose hängt immer tiefer. Aber irgendwie finden die Mädchen in deiner Klasse dich voll süss. Das geniesst du richtig. So wie Papa in deinem Alter.» ${ }^{2}$ Die Kampagne ,du.bist.deutschland.' spricht Jugendliche an und transportiert zugleich ein bestimmtes Image: ein männlicher, pubertärer Schulschwänzer, der genau weiss, dass er eigentlich zum Unterricht müsste; der Gefallen findet an der Anerkennung seiner Klassenkameradinnen und es seinem Vater gleichtut. Solange Kinder schulpflichtig sind, liegt es per Gesetz jedoch in der Verantwortung ihrer Eltern, den regelmässigen Schulbesuch zu gewährleisten. Die Absolvierung einer obligatorischen Schulzeit soll sicherstellen, dass der gesellschaftliche Nachwuchs ausgestattet mit einem notwendigen Minimum an Bildung die Schule verlässt, fähig auf eigenen Beinen zu stehen und ein autonomes Leben zu führen. Gemessen an der allgemeinen Schulpflicht sind Schlagzeilen wie «Schulschwänzen wird richtig teuer» 3 oder «Zwölfjähriger fehlte 19-mal: wird Schulschwänzen Mode?» ${ }^{4}$ umso verwunderlicher, weil es gemäss Rechtsnorm ja 
eigentlich gar keine Schulschwänzer geben dürfte. Zwar ist die Verantwortlichkeit der Eltern gesetzlich klar geregelt, doch stellt sich darüber hinaus die Frage nach den Bedingungsfaktoren für schulabsentes Verhalten nicht nur mit Blick auf den familiären Hintergrund von Schüler/innen, sondern auch auf deren schulisches Umfeld. Rumberger und Thomas (2000) etwa weisen nach, dass Schulschwänzen und vorzeitige Abgänge von der Schule einerseits auf den sozialen Hintergrund und die Komposition der Schülerschaft zurückzuführen sind, andererseits jedoch auch auf die Ressourcen einer Schule sowie deren alltägliche Prozesse und Abläufe. Coleman (1988) bezeichnet dies als soziales Kapital einer Schule. So lässt sich mit Stamm (2006) kommentieren: «Das soziale Kapital kann Schulab-sentismusaktivitäten in zweifacher Hinsicht unterstützen oder hemmen, einmal auf der Dimension der Schule (Makroebene) über Normen, Werte, Traditionen und Verhaltensrituale und zum anderen auf der Dimension der Peer- und Lehrerbeziehungen (Mikroebene), die sich in der Ausprägung von Motivations-, Volitions und Einstellungsvariablen äussert» (S. 7f.). Vor diesem Hintergrund sollen im vorliegenden Artikel familiäre Faktoren schulischen Kontextmerkmalen gegenüber gestellt und deren Bedeutung im Zusammenhang mit schwänzenden Schülern untersucht werden.

\section{Theoretische Deutungsmuster von Schulabsentis- mus}

Aus theoretischer Sicht können die Ansätze zur Beschreibung und Erklärung von Schulabsentismus in zwei Hauptströmungen unterteilt werden: die zunächst vorherrschende individuell orientierte Perspektive, welche Schulschwänzen als persönliche und allenfalls herkunftsbedingte Pflichtverletzung der Jugendlichen sah sowie die später dominierende institutionelle Betrachtungsweise, die Absentismus als Verhalten in einem bestimmten kontextuellen Setting wie Schule oder Peer-Gruppe beschreibt. Inzwischen besteht Konsens über die Notwendigkeit einer gleichzeitigen Betrachtung beider Perspektiven, wenn auch bisher im deutschsprachigen Raum erst wenige Untersuchungen vorliegen, die über eine regionale Gültigkeit hinaus aussagekräftig wären. Im folgenden Kapitel werden mögliche Deutungsmuster von Schulabsentismus vorgestellt, die entweder die Familie oder die Schule fokussieren.

\section{Die individuell-familiäre Perspektive}

Ein psychoanalytischer Blick auf Schulschwänzen widerspiegelt die in den Anfängen der Schulabsentismusforschung vorherrschende Meinung, Schulabsentismus sei ein pathologisches Verhalten, das auf Störungen der Persönlichkeit von Kindern und Jugendlichen zurückzuführen sei. Dieses "Verwahrlosungskonzept» (Oehme, 2007, S. 48) umfasst neben psychologischen auch medizinische Aspekte, nach denen Schulabsentismus als genetisch bedingte Verwahr- 
losung auch eine Reaktion des Kindes auf ein soziales Milieu und Ausdruck einer «grossen Not» (Kleist, 1930, S. 16) sein kann. Auch wenn diese Sichtweise heute kaum noch forschungsleitend zum Tragen kommt, so existieren doch Studien, welche belegen, dass schulschwänzende Kinder und Jugendliche unerwartet häufig auch an neurotischen Störungen wie Angstzuständen, extremer Traurigkeit oder Nervenschwäche leiden (Hersov \& Berg, 1980; Reid, 2005) und damit einem mikrosoziologischen Zugang mit Fokus auf familiäre Verhältnisse den Weg bereiten. In diesem Sinne kann Schulabsentismus etwa als Ausdruck eines familiären oder auch milieuspezifischen Habitus betrachtet werden, in welchem sich die Einstellung des Individuums gegenüber Schule und Schulbesuch widerspiegelt. «So entsprechen beispielsweise die Bereitschaft, sich des Bildungswesens zu bedienen, und die Möglichkeiten, dort auch Erfolg zu haben, den objektiven Bildungs- und Erfolgschancen der verschiedenen sozialen Klassen; dies wiederum ist der wichtigste Faktor für die Perpetuierung der Struktur der Bildungschancen als objektiv greifbarem Ausdruck der Relationen zwischen dem Bildungssystem und der Struktur der Klassenbeziehungen» (Bourdieu \& Passeron, 1971, S. 221). Eltern und Familie kommt hier also eine Vorbildfunktion $\mathrm{zu}$, von der die Haltung des Schulkindes gegenüber der Schule und sein Interesse am schulischen Lernen wesentlich geprägt werden. Darüber hinaus kann auch mit Bronfenbrenners (1989) sozial-ökologischem Ansatz argumentiert werden, dass in der Auseinandersetzung mit der Familie für die Jugendlichen Impulse entstehen können, welche sie zu einer Entscheidung veranlassen, die Schule mehr oder minder häufig nicht zu besuchen. Wenn etwa während der Schulzeit kein Erziehungsberechtigter im Hause ist, der bemerken könnte, dass die Tochter oder der Sohn nicht in der Schule ist, dürfte dies eher die Möglichkeit einer Schulabsenz begünstigen als wenn die Gefahr besteht entdeckt zu werden. Nach dieser individuell ausgerichteten Perspektive ist Schulabsentismus also sowohl ein selbstbestimmtes Verhalten der Jugendlichen, mit dem sie eine Entscheidung gegen eine gesellschaftliche Vorschrift treffen, als auch eine mögliche Reaktion auf familiäre Bedingungen und Situationen, die Schulschwänzen entweder notwendig oder attraktiv machen: notwendig dann, wenn etwa ein Familienmitglied Hilfe benötigt und der Jugendliche auf Initiative der Eltern zurückgehalten wird; attraktiv, wenn niemand zu Hause ist und das Schwänzen bemerken könnte. Auch verschiedene devianztheoretische Ansätze wie etwa Kontrolltheorien (Hirschi, 1969; Nye, 1958) stellen das Individuum und seine Familie ins Zentrum ihrer Betrachtung, indem etwa «ein wesentliches Moment konformen Verhaltens das Ausmass der Bindung des Individuums an die Gesellschaft ist» (Dunkake, 2007, S. 105) und damit abweichendes Verhalten Ausdruck einer mangelnden Identifikation mit gesellschaftlichen Werten und Normen. Schulabsentismus wäre in dieser Hinsicht die Nicht-Anerkennung der institutionalisierten Norm der Schulpflicht, wobei nicht nur der Schüler an sich, sondern auch dessen Familie als Primärgruppe mit sozialisatorischer Funktion im Blickfeld stehen. 


\section{Die schulisch-institutionelle Perspektive}

Strukturelle schulische Merkmale wie Schülerleistungen, Klassenwiederholung oder organisatorische Kontextbedingungen werden in Bezug auf Schulabsentismus für den deutschsprachigen Raum erstmals systematisch von Hildeschmidt (1979) erfasst. Trotz methodischer Mängel dieser rein quantitativen Untersuchung liefert sie einen ersten Hinweis darauf, dass schulische Umweltmerkmale das (unregelmässige) Schulbesuchsverhalten deutlicher zu beeinflussen scheinen als individuell-biographische und familiäre Faktoren. Im Gefolge der PISA-Studien gilt inzwischen als erwiesen, dass solche strukturellen Variablen im Sinne von Kompositions- und Institutionseffekten äusserst bedeutsam für die Bildungslaufbahn von Jugendlichen sind (Baumert, Stanat und Watermann, 2007). Bereits Durkheim (1930) geht sozialen Bedingungen für individuelles Handeln nach und versucht damit individuelles Verhalten durch den jeweiligen sozialen Kontext zu erklären. Schulen als allgemeine Bildungseinrichtung einer Gesellschaft sind ein Beispiel solcher institutionalisierter Kontexte, mit denen das Verhalten ihrer Mitglieder interagiert. Schulabsentismus wäre unter diesem Aspekt der Ausdruck einer «mangelnden Passung zwischen den Egoismen der Individuen und dem allgemeinen Interesse des Staates» (Kurtz, 2007, S. 235). Parsons (1960) nimmt Ende der 50er Jahre noch expliziter als Durkheim Bezug zur Relevanz schulischer Kontexte auf das Verhalten ihrer Schüler, indem er Schulklassen als soziale Systeme bezeichnet, die sich in einer Tauschbeziehung mit anderen, nicht sozialen Systemen befinden (S. 238). Die Schulklasse steht demnach für Parsons mit dem kulturell bestimmten System von Bildungsinhalten und -zielen sowie mit den individuellen Persönlichkeitssystemen der Klassenmitglieder in einem Austauschverhältnis (Kooperation): "This cooperation cannot always be taken for granted; it has to be motivated. Witness, for example, the problem of truancy in schools, to say nothing of passive resistance to learning...Since sheer coercion is not adequate, service-performer must offer something to induce adequate cooperation» (S. 72f.). Schule ist ein Ort, an dem gemeinsam Zeit verbracht wird und in dem Beziehungen zwischen Schüler/innen und Lehrpersonen entstehen, deren Valenz letztlich als gelingend oder nicht gelingend wahrgenommen wird. Schulabsentismus ist somit auch ein Ausdruck einer nicht ausreichend gelingenden Beziehungsstruktur in der Schule, wie auch Stamm (2006) und Moore (2004) zeigen konnten. Gesamthaft ist Schulabsentismus zwar stets ein Verhalten, dass von Individuen gezeigt wird, dennoch spielt sich dieses Verhalten immer auch in Bezug auf ein schulisches Setting ab. Nach Stamm (2006) erklärt dies, "warum Schülerinnen und Schüler, die mit Mitschülern und Lehrerschaft nicht klar kommen, den Unterricht rein aus sozialen Gründen schwänzen [...] und auch dann schulabsent bleiben, wenn sie Fördermassnahmen im Sinne eines externen Coachings bekommen. Sie bleiben überzeugt, dass ihre Präsenz an der Schule keine vorrangige Bedeutung hat, die Lehrpersonen sich nicht für sie interessieren und ihnen auch nicht helfen wollen» (a.a.O., S. 8). 
Beide dargestellten theoretischen Deutungsmuster werden im folgenden Kapitel zum Forschungsstand belegt und vertieft.

\section{Forschungsstand und Fragestellung}

Vornehmlich im anglo-amerikanischen Raum existieren Studien, die sowohl die individuelle als auch die institutionelle Perspektive berücksichtigen und erfassen. Aber auch in Kontinentaleuropa liegen dazu inzwischen mehrere Studien vor, wenn auch zumeist in regional begrenztem Umfang und mit Schulversäumnissen als Nebeninteresse (Goethe, 2005; Kraus, Heppekausen, Barrera \& Orth, 2004; Oberwittler, Blank, Köllisch \& Naplava, 2001; Wilmers et al., 2002;). Gemäss der Sekundäranalyse von Weiss (2007) ist die Varianz der Befunde, wie häufig geschwänzt wird, in verschiedenen deutschen Studien beträchtlich: «Je nach Untersuchung schwänzen zwischen $10 \%$ und fast $60 \%$ der Jugendlichen die Schule» (S. 44), wonach Schulabsentismus in der Tat keine Randerscheinung ist (Stamm, 2006). Die Familie als Kontext devianten Verhaltens wird soziologisch vornehmlich im Rahmen von Kontrolltheorien aufgegriffen (Dunkake, 2007; Hirschi, 1969; Nye, 1958). Unterschieden werden dabei strukturelle Merkmale wie der sozio-ökonomische Status, die Zahl der Geschwister, die Anwesenheit eines oder beider Elternteile im Haushalt oder der Familienstand der Eltern von so genannten innerfamiliären Komponenten wie der Qualität der Beziehung zwischen Eltern und Kindern oder der gemeinsam verbrachten Zeit. Dunkake (2007) kann in ihrer Analyse zur Bedeutung familialer Kontrolle für schulmeidendes Verhalten zeigen, dass eine negative emotionale Bindung der Kinder an ihre Eltern sowie eine niedrige elterliche Kontrolle das Risiko der Schulverweigerung direkt erhöhen. Bildungsrelevante Abläufe und Entscheidungen in Familien sind enorm schwierig zu untersuchen (Fend, 2006), etwa wie über weiterführende Bildungswege diskutiert und nachgedacht oder wie die Entscheidung für eine bestimmte Schule getroffen wird. Die Annäherung an die Wirkungen der Familie erfolgt daher häufig über die Trennung schulischer Faktoren von Herkunftsmerkmalen. So konnten etwa Rumberger und Thomas (2000) zeigen, dass das von Coleman (1988) als zentral identifizierte soziale Kapital einer Schule in Form von Normen, Werten oder Ritualen (Makroebene Schule) sowie als Beziehungen, Einstellungen oder Motivation (Mikroebene Schulangehörige) die Identifikation der Schülerschaft mit ihrer Schule wesentlich beeinflusst. Coleman (1987) war es auch, der den Blick von der Familie auf die Schule lenkte. Er baute seine Untersuchung zu Anwesenheits- und Schulabbrecherraten und damit verbundenen schulischen Faktoren auf soziologischen Überlegungen auf und beschrieb Schulen als gegenüber den Familien formal komplementäre Institutionen, die bei Kindern aus hohen Bildungsschichten einen stärkeren Einfluss nehmen können als bei Kindern aus niedrigeren Schichten. Letzten Endes wirkt jedoch nicht nur die Schule an sich als 
differenzielles Lern- und Entwicklungsmilieu, sondern die Verbindung zwischen Schule und Elternhaus. Die Schule liefert dabei einen Teil der Komponenten sozialen Kapitals für die Sozialisierung der Schüler (Lerngelegenheiten, Anforderungen und Anreize), während die Familie als primäres Umfeld des Kindes weitere Faktoren beisteuert (Einstellungen, Arbeitshaltung/Anstrengung und Selbstkonzept). Schulbezogene Zusammenhänge mit Absenzen wurden vielfach gefunden, so etwa, dass in anforderungsniedrigen Schulniveaus signifikant seltener geschwänzt wird als in anspruchsvollen Schulformen (Schreiber-Kittl \& Schröpfer, 2002; Weissbrodt, 2007). In der Schweizer Grundlagenstudie von Stamm et al. (2007) zeigte sich hier jedoch ein entgegengesetztes Bild: Schüler/ innen der erweiterten Schulzüge schwänzten häufiger als die Jugendlichen im grundlegenden Schulniveau. Darüber hinaus gilt als unbestritten, dass eine positive Beziehung zu den Lehrpersonen sowie das Klassenklima negativ mit Absentismus korrelieren (Fend, Knörzer, Nagl, Specht \& Väth-Szusdziara, 1976; Reynolds, Jones \& St. Leger, 1976; Tillmann, Holler-Nowitzki, Holtappels, Meier \& Popp, 1999).

Gemäss den einschlägigen Forschungen zu Schulabsentismus ist davon auszugehen, dass Schulschwänzen mit einem Bedingungsgeflecht aus individuellen Eigenschaften und Situationsmerkmalen aus Familie und Schule in Zusammenhang steht. Hier soll daher untersucht werden, inwieweit unter Kontrolle individueller Merkmale bestimmte familiale und schulische Charakteristika relevant für die Häufigkeit von Absenzen sind. Die dahinter stehende Fragestellung, inwieweit das familiäre oder das schulische Umfeld erklärungsstark für Absentismus sind, soll anhand von Mehrebenenanalysen beantwortet werden.

\section{Methode}

Die Daten werden in mehrebenenanalytischen Modellen in der Software MLwiN analysiert. Blockweise werden Variablengruppen als Prädiktoren der multiplen Regressionen in die Berechnungen einbezogen, so dass das Modell sukzessive spezifiziert wird. Die genannten Kompositionsmerkmale bilden das erste Modell, die familienbezogenen Variablen kommen im zweiten Modell hinzu und im dritten Modell werden sowohl die strukturellen Merkmale der Schule als auch die Schülerurteile berücksichtigt. Fehlende Werte werden im für die Mehrebenenanalysen verwendeten Programm MLwiN durch multiple Imputation ersetzt (MCMC-Verfahren zur Schätzung fehlender Werte, 5 Imputationen). Für die Konvergenzdiagnostik des MCMC-Schätzverfahrens wurden die Traceplots für den Mittelwert und einen fehlenden Wert berücksichtigt sowie die Autokorrelationsfunktion des Mittelwerts und so die Plausibilität der geschätzten, imputierten Werte gesichert. 


\section{Studie}

\section{Stichprobe und Datensatz}

Die hier analysierten Daten entstammen der Schweizer Grundlagenstudie von Stamm et al. (2007) und repräsentieren 3942 Schülerinnen und Schüler der deutschsprachigen Schweiz. Anhand standardisierter Fragebögen wurden im April 2006 die Schüler in 239 Klassen bzw. 28 für die Deutschschweiz repräsentativen Schulen befragt sowie deren Klassenlehrpersonen und Schulleiter/innen. Die Schüler/innen besuchten zum Befragungszeitpunkt die Klassen sieben bis neun, wobei jeweils ein Drittel der Stichprobe auf die einzelnen Jahrgangsstufen entfällt. Die Schüler gingen zu 58\% auf eine Schule des erweiterten Niveaus, 34\% auf eine Schule des grundlegenden Niveaus und 8\% besuchten eine Kleinklasse. Ihr Alter variiert zwischen 12 und 16 Jahren, durchschnittlich waren die teilnehmenden Schüler zum Zeitpunkt der Befragung 14,5 Jahre alt $(s d=1.2)$. Die Verteilung der Geschlechter auf die Stichprobe kann als ausgeglichen bezeichnet werden: $50,6 \%$ sind männlichen, $49,4 \%$ weiblichen Geschlechts. 71\% der befragten Schülerinnen und Schüler sind Schweizerischer Staatsangehörigkeit, 29\% haben einen ausländischen Pass. Für die hier vorgelegte Teilstudie wurden lediglich Schüler/innen aus Regelklassen berücksichtigt $(n=3668)$, da die Kleinklassenschüler/innen teilweise mit gegenüber den regulären Skalen reduzierten Fragesets untersucht wurden, was die Vergleichbarkeit deutlich begrenzen würde.

\section{Variablen und Erhebungsinstrumente}

Die in dieser Analyse verwendeten Variablen wurden anhand eines standardisierten Schülerfragebogens erhoben. Die abhängige Variable ist ein gewichteter Häufigkeitsindex, welcher das Ausmass der durch Schwänzen versäumten Unterrichtszeit im laufenden Schuljahr widerspiegelt. Differenziert wurde zwischen dem Fehlen einzelner Stunden («Hast du in diesem Schuljahr schon.... in bestimmten Fächern/Randstunden/eine Prüfung geschwänzt?»), halber Tage («... Halbtage geschwänzt?») sowie ganzer und mehrerer aufeinander folgender Tage («...einzelne Tage/mehr als 2 Tage hintereinander geschwänzt?»). Die Schüler/ innen hatten jeweils die Auswahl zwischen den Kategorien ,noch nie', ,ab und zu' und ,mehr als fünfmal'.

Als unabhängige Variablen wurden zunächst mögliche Kompositionsmerkmale der Schüler/innen bestimmt, die zur validen Identifikation von Familienund Schuleffekten in der Modellspezifizierung kontrolliert werden mussten (Baumert et al., 2007). Diese Individualmerkmale sind das Geschlecht, der sozio-ökonomische Status (ISEI nach Ganzeboom \& Treiman, 1996), die Mathematiknote als Leistungsindikator ${ }^{5}$, Klassenwiederholung, die Jahrgangsstufe sowie das Anforderungsniveau der Schule. Der sozio-ökonomische Status wurde anhand der aktuellen Beschäftigung beider Elternteile erhoben und operationalisiert, indem der jeweils höhere Wert (Vater oder Mutter) innerhalb 
einer Familie als statusrelevant ausgewählt wurde. Das Geschlecht und die Klassenwiederholung ( $\mathrm{ja} / \mathrm{nein}$ ) gingen als separat kodierte Dummy-Variablen in die Analysen ein, Jahrgangsstufe und Anforderungsniveau als kontrastkodierte Dummy-Variablen mit den Referenzkategorien ,8. Schuljahr' und ,erweitertes Niveau'. Die Mathematiknote wurde als kontinuierliche Variable eingesetzt und um den Mittelwert zentriert (grand-mean; vgl. Lüdtke et al., eingereicht). Über diese Kompositionsmerkmale hinaus wurden als strukturelle Kontextmerkmale die Schul- und Klassengrösse und auf individueller Ebene sowohl familien- als auch schulbezogene Skalen erfasst. Die Schulgrösse wurde in drei Stufen unterteilt, wobei Schulen mit bis zu 100 Schüler/innen als ,klein' klassifiziert wurden, mit 101-200 Schülern als ,mittel' und mit mehr als 200 als ,gross'. Die Skalen mit Bezug zur Familie entstammen den Studien von Eder (1998), Roth (2002)

$\begin{array}{lll}\begin{array}{l}\text { Punktwert auf der Skala, } \\ \text { Häufigkeit der Absenzen' } \\ \text { (gewichtet) }\end{array} & \text { Bezeichnung } & \text { Herleitung } \\ 0 & \text { Nicht-Schwänzer } & \begin{array}{l}\text { Schüler/innen, die im zum Zeitpunkt der Befragung } \\ \text { laufenden Schuljahr noch nicht geschwänzt hatten }\end{array} \\ 1-6 & \begin{array}{l}\text { Gelegentliche } \\ \text { Schwänzer } \\ \text { Schwäler/innen, die maximal einzelne Schulstunden } \\ \text { fehlen, nicht jedoch halbe oder ganze Tage }\end{array} \\ \text { Blockschwänzer } & \begin{array}{l}\text { Innerhalb dieser Gruppe herrscht die grösste Hete- } \\ \text { rogenität vor, da in diesem Fall die Zusammensetzung } \\ \text { der Punkte rechnerisch auf verschiedene Weise zu- } \\ \text { stande kommen kann: aufgrund mehrerer versäumter } \\ \text { Einzelstunden und eines einzigen Halbtages, oder aber } \\ \text { auf der Basis vieler gefehlter halber und ganzer Tage, } \\ \text { zu denen einzelne Stunden hinzukommen. } \\ \text { Schüler/innen, welche am häufigsten die Schule } \\ \text { schwänzen, erzielen Werte von 19 Punkten und mehr, } \\ \text { wobei in diesem Fall auch mehr als zwei Tage hinte- } \\ \text { reinander geschwänzt wurde. }\end{array} \\ & \begin{array}{l}\text { Massive } \\ \text { Blockschwänzer }\end{array} \\ & \end{array}$

und Kittl et al. (2005). Das Familienklima wurde anhand zweier Dimensionen operationalisiert: die Kontrolle seitens der Eltern ( genau festgelegt, was man darf und was nicht»; 4 Items, Cronbach's $\alpha=.73$ ) sowie die aktive Freizeitgestaltung («Wir unternehmen an jedem Wochenende etwas ausser Haus»; 4 Items, Cronbach's $\alpha=.65$ ). Als zweite familienrelevante Skala wurde der Erziehungsstil erfragt, wobei zwei Dimensionen unterschieden wurden: Elterliche Zuwendung («Bei Problemen in der Schule halten meine Eltern auf jeden Fall zu mir»; 3 Items, Cronbach's $\alpha=.65$ ) und schulbezogenes Monitoring ( $«$ Meine Eltern fragen oft, was ich in der Schule gemacht oder gelernt habe»; 4 Items, Cronbach's $\alpha=.61$ ). Die Schülerurteile über Merkmale ihrer Klasse und Schule wurden von Eder (1998), Ditton \& Merz (1999) und Kittl et al. (2005) übernommen. Die Klassengemeinschaft wurde über 5 Items erfragt ( Wenn jemand aus meiner Klasse Hilfe braucht, helfen wir einander gerne», Cronbach's $\alpha=.61$ ). Ausserdem wurde das Schülerurteil über die persönliche Beziehung zu ihren Lehrpersonen erhoben («Meistens werde ich von den 
Lehrpersonen fair behandelt», 5 Items, Cronbach's $\alpha=.80)$. Das Schulklima wurde anhand von Items der Studien von Eder (1998) und Kittl et al. (2005)

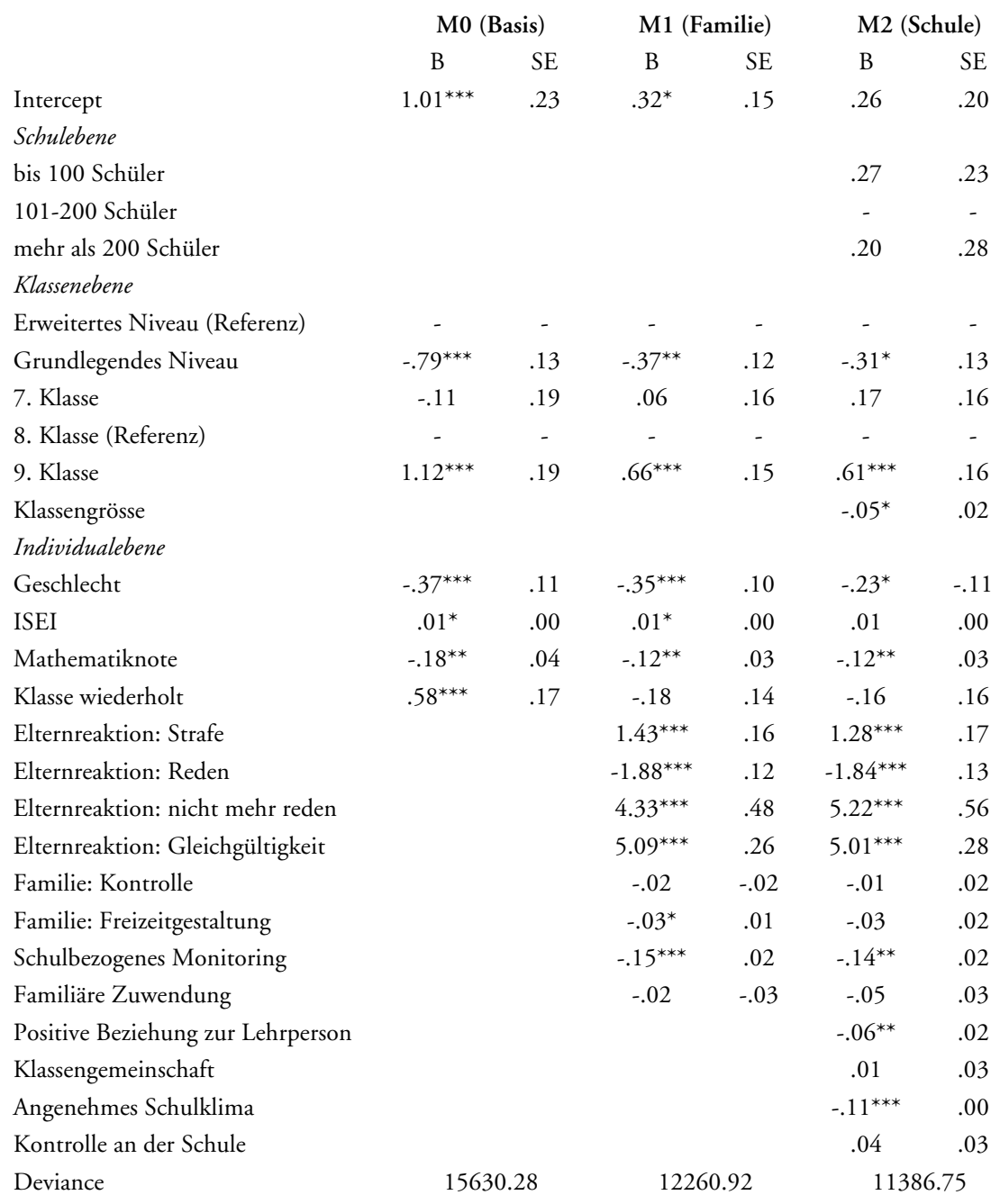

erfasst («Die Stimmung an unserer Schule ist meistens: heiter, fröhlich/ein wenig fröhlich/ein wenig gedrückt/gedrückt, lustlos», 4 Items, Cronbach's $\alpha=.61$ ). Schliesslich die Strenge und Kontrolle im Schulhaus wurde mittels einer Skala von Eder (1998) erfragt («Die Lehrpersonen achten darauf, dass wir die Hausordnung/Schulordnung einhalten», 4 Items, Cronbach's $\alpha=.68$ ).

Kontextmerkmale sind damit entweder strukturelle Merkmale (Klassen- und Schulgrösse), welche nicht aggregiert zu werden brauchten oder aber Schülerurteile einer geteilten sozialen Wirklichkeit, deren Aggregierung inhaltlich nicht 
notwendig ist. Die individuelle Entscheidung zu schwänzen oder nicht wird stärker der individuellen Wahrnehmung schulischer Aspekte zugeschrieben als konstruierten Durchschnittswerten, so dass diese Prädiktoren auf der individuellen Ebene einbezogen werden. Die Prädiktoren auf Individualebene werden im Hinblick auf die Interpretation der Koeffizienten um den Mittelwert (grandmean; vgl. Lüdtke et al., 2009) zentriert.

\section{Ergebnisse}

In Tabelle 2 wird ersichtlich, dass eine ganze Reihe von Merkmalen der Schüler/ innen, ihrer Familie und ihrer Einschätzung der Schule zusammen wirkt, wenn die Jugendlichen die Schule schwänzen. Die Intercepts sind jeweils so zu lesen, dass in einer Schule, welche auf allen im Modell enthaltenen Variablen durchschnittliche Werte aufweist, ein Absenzen-Wert in der Höhe des Intercepts erreicht wird. Demnach sind die Koeffizienten stets in Bezug auf das Intercept zu lesen und zu addieren. Gedanklich kann die Skala ,Häufigkeit der Absenzen' in vier Gruppen unterteilt werden:

Tabelle 1: Einteilung der Skala, Häufigkeit der Absenzen' in vier Stufen Auf dieser Basis werden in der folgenden Tabelle 2 die drei blockweise konstruierten Modelle vorgestellt. 
Tabelle 2: Individuelle, familiäre und schulische Wirkungsfaktoren bei Schulabsentismus

$\mathrm{N}=3668$; Signifikanzniveaus: ${ }^{* *}$ : p<.001; **: p<.01; *:p<.05.

Modell M0 als Basismodell zeigt auf, inwieweit bereits so genannte Kompositionsmerkmale der Schüler Unterschiede bezüglich der Häufigkeit ihrer Absenzen vom Unterricht erklären können. Alle sechs einbezogenen Variablen tragen signifikant dazu bei, diese Unterschiedlichkeit zu erläutern: am stärksten relevant ist das Alter resp. die Jahrgangsstufe der Jugendlichen $(\mathrm{B}=1.12)$. Auch das Schulniveau spielt eine bedeutende Rolle, wenn man lediglich die Regelschüler in Bezug auf Ihr Anforderungsniveau vergleicht (grundlegend vs. erweitert; $\mathrm{B}=-.79$ ): im grundlegenden Niveau wird signifikant häufiger geschwänzt als in Schulzügen mit erweiterten Ansprüchen. Eine Klassenwiederholung scheint deutlich mit der Häufigkeit von Absenzen zusammenzuhängen $(B=.58)$ und Mädchen schwänzen in dieser Stichprobe seltener als ihre männlichen Klassenkameraden $(\mathrm{B}=-.37)$. Schüler/innen mit einer guten Mathematiknote schwänzen seltener als Mitschüler mit einer schwächeren Note $(\mathrm{B}=-.18)$. Ein schwach signifikanter Einfluss zeigt sich auch für den sozio-ökonomischen Status der Jugendlichen $(\mathrm{B}=.01)$, tendenziell schwänzen also Jugendliche mit einem höheren Status häufiger als andere.

In Modell M1 wurden zusätzlich zu den Variablen des Basismodells die familienbezogenen Prädiktoren in die Analyse eingefügt. Der frappierendste Befund in diesem Modell ist, dass die Klassenwiederholung gegenüber dem Basismodell ihren Einfluss auf das Schulschwänzen verliert, wenn die familiären Merkmale mit betrachtet werden. Alle übrigen Kompositionsmerkmale bleiben signifikant. Die mit Abstand grösste Effektstärke weisen die Elternreaktionen auf Schulschwänzen auf: reagieren die Eltern gleichgültig auf Absentismus $(B=5.09)$ oder reden sie nicht mehr mit ihrem Kind $(B=4.33)$, so scheint umso häufiger geschwänzt zu werden. Sprechen sie hingegen nach einer unerlaubten Absenz mit den Jugendlichen darüber $(B=-1.88)$, schwänzen die Schüler/innen seltener. Strafen als Sanktion von Absentismus $(B=1.43)$ zeigen bei den Jugendlichen einen verstärkenden Effekt auf Absenzen: reagieren die Eltern mit Strafe, so wird allem Anschein nach das Gegenteil vom angestrebten Verhalten erreicht. Darüber hinaus erwies sich das schulbezogene Monitoring der Eltern als bedeutsam für Schulabsentismus ( $\mathrm{B}=-.15)$; das Interesse der Familienmitglieder für das in der Schule Gelernte scheint unerlaubte Absenzen zu hemmen. Für familiäre Kontrolle und Zuwendung hingegen konnten keine wesentlichen Effekte nachgewiesen werden.

In Modell M2 schliesslich kommen noch die schulbezogenen Variablen hinzu (strukturell und Schülerurteile). Wie in Modell M1 zeigt die Wiederholung einer Klasse keine relevanten Effekte mehr; auch der sozio-ökonomische Status hat in dieser Kombination keine Erklärungskraft mehr für Absenzen. Die übrigen Variablen des Basismodells bleiben signifikant. Von den beiden struk- 
turellen Schulmerkmalen Schul- und Klassengrösse konnte in dieser Modellzusammensetzung lediglich für die Klassengrösse ein signifkanter Effekt gefunden werden $(\mathrm{B}=-.05)$ : Je grösser die Klasse ist, desto weniger wird geschwänzt. Die Schülerurteile bezüglich der Beziehungen im Klassenzimmer zeigten sich nur teilweise als bedeutsam für die Unterschiedlichkeit der Absenzen: relevant sind die Beziehung zu den Lehrpersonen, wie sie von den Schüler/innen empfunden wird $(\mathrm{B}=-.06)$ und das Schulklima $(\mathrm{B}=-.11)$. Weder die Klassengemeinschaft noch die Kontrolle von Regeln an der Schule zeigen signifikante Zusammenhänge mit der Häufigkeit geschwänzter Schulstunden. 


\section{Diskussion}

Mit diesem Beitrag sollte der Frage nachgegangen werden, inwieweit neben individuellen Merkmalen von Jugendlichen deren Familie und Schule eine Rolle spielen, wenn es um die Häufigkeit von Schulschwänzen geht. Den Effektstärken nach ist es eindeutig die Familie, die den bedeutsamsten Einfluss auf die Absenzenhäufigkeit der Jugendlichen nimmt. Bereits sozio-demographische Merkmale der Jugendlichen tragen zur Erklärung bei, weshalb Schulschwänzen einerseits ein verbreitetes Phänomen ist und andererseits in so heterogenem Ausmass vorliegt. Gegenüber einem Durchschnittsjugendlichen (Variablen des Basismodells) schwänzt also ein männlicher Neuntklässler, der bereits eine Klasse wiederholt und eine schlechte Note in Mathematik sowie einen relativ hohen sozio-ökonomischen Status hat, signifikant häufiger. Betrachtet man darüber hinaus familiäre Merkmale, so verändert sich das Zusammenspiel des Bedingungsgeflechts für Schulabsentismus merklich: Klassenwiederholung ist nicht mehr erklärungsstark, sehr wohl jedoch die Reaktionen der Eltern. Gleichgültigkeit, Strafen oder nicht mehr mit dem Kind sprechen sind Sanktionen, die einen sehr starken verstärkenden Effekt haben: reagieren die Eltern mit derart ablehnenden Verhaltensweisen, so steigt die Absentismushäufigkeit an wie bei keinem anderen untersuchten Merkmal. Dies entspricht den Befunden von Dunkake (2007). Aber auch weniger direkt auf das Schulschwänzen bezogene Familienmerkmale wie gemeinsame Freizeitgestaltung oder das Interesse der Eltern für schulische Erlebnisse und Erfahrungen ihrer Kinder (Monitoring) erwiesen sich als bedeutsam für das Absentismusverhalten, was auch Coleman (1988) mit seinem Begriff des sozialen Kapitals beschreibt.

Auf strukturell-familiäre Merkmale wurde in diesem Artikel verzichtet, da der Fokus sowohl bei den schulischen als auch bei den familiären Variablen auf den Schülerurteilen lag. Auch die Perspektive der Lehrpersonen und Schulleiter hatte keinen Platz und könnte das Bild der Bedingungsfaktoren noch verdichten. Kausale Aussagen sind auf der Basis einer Querschnittsuntersuchung nicht möglich, weshalb die Effektstärken in diesem Artikel als Zusammenhänge mit und nicht als direkte Ursachen für Absentismus zu sehen sind. Dennoch konnte einmal mehr die inzwischen gängige Erkenntnis bestätigt werden, dass Schulabsentismus ein multifaktoriell bedingtes jugendtypisches Verhalten ist, welches weder rein auf strukturelle Merkmale noch auf einzelne Persönlichkeitsfaktoren zurückgeführt werden kann. Ob und wie häufig eine Schülerin oder ein Schüler dem Unterricht unerlaubt fernbleibt, beruht immer wieder neu auf einer situativ einzigartigen Entscheidung. Die Gelegenheitsstruktur dieser Entscheidung wird beeinflusst von Sozialisationserfahrungen, die die Jugendlichen gemacht haben und von ihrer Einstellung gegenüber ihrer Schule. Auch Schüler/innen, die sich wohlfühlen und gerne zur Schule gehen, schwänzen ab und an den Unterricht. Es kommt jedoch darauf an, anhand der hier identifizierten Bedingungsmuster für gehäuftes Schwänzen die Risikofaktoren zu erkennen, welche einen Jugend- 
lichen zu gehäuftem Absentismus führen können. In der vorliegenden Analyse scheinen die innerfamiliären Merkmale die gewichtigste Rolle für das Ausmass des Absentismus zu spielen, wobei neben den persönlichen Eigenschaften auch schulische Faktoren von Bedeutung sind. Interesse am Jugendlichen seitens der Familie und der Schule scheinen dem regelmässigen Schulbesuch gut zu tun, wobei Schulschwänzen auf diese Weise sicherlich nicht zu verhindern ist. Dies ist jedoch auch nicht notwendig, wenn gemässigt geschwänzt wird und keine Gefahr besteht, dass die Schüler/innen sich durch ihre Absenzen nachhaltig vom institutionalisierten Bildungsprozess abkoppeln. Zentral bei der Analyse von Bedingungsmustern des Schulschwänzens ist das Erkennen des Übergangs vom gelegentlichen zum blockweisen und massiven Schwänzen, denn hier kann und muss pädagogisch angesetzt werden, wenn aktiv gegen nachhaltig schädigenden Absentismus vorgegangen werden soll. In dieser Studie machen eindeutig die Elternreaktionen den Unterschied zwischen gelegentlichem und teilweise ,schwachem Blockschwänzen' aus, gemeinsam mit schwächer wirkenden anderen Faktoren. Die Einstellung gegenüber der Schule, die ein Kind vom Elternhaus mitbekommt, scheint demnach ein wichtiger Schlüsselfaktor zur Prävention von massivem Absentismus zu sein.

\section{Anmerkungen}

1 Die vorliegende Studie wurde anhand von Daten aus einem mit Mitteln des Schweizerischen Nationalfonds zur Förderung der wissenschaftlichen Forschung unterstützten Projekt (Projekt Nr. 100013-107961/1) durchgeführt. Die Projektleitung hatte Prof. Dr. Margrit Stamm inne.

2 Anzeigentext in der Süddeutschen Zeitung vom 4. Februar 2008.

3 Der Titel entstammt dem WDR-Streiflicht «Brennpunkt Schule» vom 24.Mai 2006. http:// www.wdr.de/themen/wissen/bildung/brennpunkt_schule/bussgeld_schulschwaenzer/index.jhtml? rubrikenstyle=wissen \&rubrikenstyle=brennpunkt_schule

4 Mainpost vom 25.November 2007.

5 Zusätzlich zur Mathematiknote lag auch die Deutschnote als Leistungsindikator vor. Die Zerlegung der Varianz auf Klassen- und Individualebene (ICC) zeigte jedoch, dass mehr als 99\% der Varianz auf Schülerebene erklärt werden. Zusätzlich ist die Varianz der Deutschnote in der gesamten Stichprobe sehr gering. Daher wurde ausschliesslich die Mathematiknote in die Modellierung einbezogen.

\section{Literatur}

Baumert, J., Stanat, P. \& Watermann, R. (2006): Schulstruktur und die Entstehung differenzieller Lern- und Entwicklungsmilieus. In J. Baumert, P. Stanat \& R. Watermann (Hrsg.), Herkunftsbedingte Disparitäten im Bildungswesen. Vertiefende Analysen im Rahmen von PISA 2000 (S. 95-188). Wiesbaden: VS Verlag für Sozialwissenschaften.

Bourdieu, P. \& Passeron, J.-C. (1971). Die Illusion der Chancengleichheit. Stuttgart: Ernst Klett.

Bronfenbrenner, U. (1989). Die Ökologie der menschlichen Entwicklung. Natürliche und geplante Experimente. 5., vollständig überarbeitete Auflage. Weinheim: Beltz.

Coleman, J. S. (1987). Families and Schools. Educational Researcher, 16 (6) 32-38. 
Coleman, J. S. (1988). Social Capital in the Creation of Human Capital. American Journal of Sociology, 94, 95-120.

Ditton, H. \& Merz, D. (1999). DfG-Projekt: Qualität von Schule und Unterricht. Universität Osnabrück.

Dunkake, I. (2007): Schulverweigerung: Eine Folge mangelnder familaler Kontrolle? In M. Wagner (Hrsg.), Schulabsentismus. Soziologische Analysen zum Einfluss von Familie, Schule und Freundeskreis (S. 105-138). Weinheim und München: Juventa.

Durkheim, E. (1930). Le suicide. Etude de sociologie. Paris: Felix Alcan.

Eder, F. (1998). Linzer Fragebogen zum Schul- und Klassenklima für die 8.-13. Klasse (LFSK 8-13). Handanweisung. Göttingen: Hogrefe.

Fend, H. (2006). Neue Theorie der Schule. Einführung in das Verstehen von Bildungssystemen. Wiesbaden: VS Verlag für Sozialwissenschaften.

Fend, H., Knörzer, W., Nagl, W., Specht, W. \& Väth-Szusdziara, R. (1976). Sozialisationseffekte der Schule. Weinheim: Beltz.

Ganzeboom, H.B.G. \& Treiman, D.J. (1996). Internationally Comparable Measures of Occupational Status for the 1988 International Standard Classification of Occupations. Social Science Research, 25, 201-239.

Goethe, F. (2005). Zum Ausmaß des Schulschwänzens - Eine Darstellung der neueren empirischen Untersuchungen und ihrer Methoden. In G. Barth \& J. Henseler (Hrsg.), Jugendliche in Krisen. Über den pädagogischen Umgang mit Schulverweigerern. Baltmannsweiler: Schneider Verlag Hohengehren.

Hersov, L. \& Berg, I. (Eds.) (1980). Out of School. Modern Perspectives in Truancy and School Refusal. Oxford: Pergamon Press.

Hildeschmidt, A. (1979). Verbreitung und Bedingungen unregelmässigen Schulbesuchs. In A. Hildeschmidt et al., Unregelmässiger Schulbesuch. Verbreitung, Bedingungen, Interventionsmöglichkeiten. Weinheim und Basel: Beltz, S. 84-110.

Hirschi, T. (1969). Causes of Delinquency. Berkeley: University of California Press.

Kittl, H., Mayr, A., Schiffer, B. (2005). Early School Leaving and Drop out. Zwischenbericht 2005. Graz: Institut für Erziehungswissenschaften.

Kleist, F. (1930). Was geht uns der Schulschwänzer an? In P. Oesterreich (Hrsg.), Die Neue Erziehung. Monatsschrift für entschiedene Schulreform und freiheitliche Schulpolitik. XII. Jahrgang (o. Heftnummer). Jena: Verlag Karl Zwing, 9-17.

Kraus, L., Heppekausen, K., Barrera, A. \& Orth, B. (2004). Die Europäische Schülerstudie zu Alkohol und anderen Drogen (ESPAD): Befragung von Schülerinnen und Schülern der 9. und 10. Klasse in Bayern, Berlin, Brandenburg, Hessen, Mecklenburg-Vorpommern und Thüringen, IFT-Berichte Bd. 141. München: IFT Institut für Therapieforschung.

Kurtz, T. (2007). Bildung und Erziehung in der soziologischen Theorie. Zeitschrift für Erziehungswissenschaft, 2, 213-249.

Lüdtke, O., Robitzsch, A., Trautwein, U. \& Kunter, M. (2009). Assessing the Impact of Learning Environments: How to Use Student Ratings in Multilevel Modelling. Contemporary Educational Psychology, 34, 120-131.

Moore, D. (2004). Bigger or better? School Planning and Management, 5, 67-69. Dayton: Peter Li.

Nye, F. I. (1958). Family Relationships and Delinquent Behavior. New York: John Wiley.

Oberwittler, D., Blank, T., Köllisch, T. \& Naplava, T. (2001). Soziale Lebenslagen und Delinquenz von Jugendlichen. Ergebnisse der MPI-Schulbefragung 1999 in Freiburg und Köln. Arbeitsbericht 1/2001 aus dem Max-Planck-Institut für ausländisches und internationales Strafrecht. Freiburg i. Br.: edition iuscrim.

Oehme, A. (2007). Schulverweigerung. Subjektive Theorien von Jugendlichen zu den Bedingungen ihres Schulabsentismus. Hamburg: Verlag Dr. Kovac.

Parsons, T. (1960). Structure and Process in Modern Societies. Illinois: The Free Press of Glencoe. 